\title{
Alteration of renal respiratory Complex-III during experimental
} type-I diabetes

\author{
Shankar Munusamy ${ }^{1}$, Hamida Saba ${ }^{1}$, Tanecia Mitchell ${ }^{1}$, Judit K Megyesi², \\ Robert W Brock ${ }^{3}$ and Lee Ann MacMillan-Crow*1
}

\begin{abstract}
Address: ${ }^{1}$ Department of Pharmacology \& Toxicology, Division of Nephrology, College of Medicine, University of Arkansas for Medical Sciences, Little Rock, AR 72205, USA, ${ }^{2}$ Department of Internal Medicine, Division of Nephrology, College of Medicine, University of Arkansas for Medical Sciences, Little Rock, AR 72205, USA and ${ }^{3}$ Department of Physiology \& Pharmacology and Center for Interdisciplinary Research in Cardiovascular Sciences, West Virginia University School of Medicine Morgantown, WV 26506, USA

Email: Shankar Munusamy - munusamyshankar@uams.edu; Hamida Saba - sabahamida@uams.edu; Tanecia Mitchell - tmitchell@uams.edu; Judit K Megyesi - megyesijuditk@uams.edu; Robert W Brock - rbrock@ hsc.wvu.edu; Lee Ann MacMillan-Crow* - lmcrow@uams.edu

* Corresponding author
\end{abstract}

Published: 23 January 2009

BMC Endocrine Disorders 2009, 9:2 doi:10.1/86/1472-6823-9-2

This article is available from: http://www.biomedcentral.com/l472-6823/9/2

(C) 2009 Munusamy et al; licensee BioMed Central Ltd.

This is an Open Access article distributed under the terms of the Creative Commons Attribution License (http://creativecommons.org/licenses/by/2.0), which permits unrestricted use, distribution, and reproduction in any medium, provided the original work is properly cited.
Received: 12 September 2008

Accepted: 23 January 2009

\begin{abstract}
Background: Diabetes has become the single most common cause for end-stage renal disease in the United States. It has been established that mitochondrial damage occurs during diabetes; however, little is known about what initiates mitochondrial injury and oxidant production during the early stages of diabetes. Inactivation of mitochondrial respiratory complexes or alteration of their critical subunits can lead to generation of mitochondrial oxidants, mitochondrial damage, and organ injury. Thus, one goal of this study was to determine the status of mitochondrial respiratory complexes in the rat kidney during the early stages of diabetes (5-weeks post streptozotocin injection).
\end{abstract}

Methods: Mitochondrial complex activity assays, blue native gel electrophoresis (BN-PAGE), Complex III immunoprecipitation, and an ATP assay were performed to examine the effects of diabetes on the status of respiratory complexes and energy levels in renal mitochondria. Creatinine clearance and urine albumin excretion were measured to assess the status of renal function in our model.

Results: Interestingly, of all four respiratory complexes only cytochrome c reductase (ComplexIII) activity was significantly decreased, whereas two Complex III subunits, Core 2 protein and Rieske protein, were up regulated in the diabetic renal mitochondria. The BN-PAGE data suggested that Complex III failed to assemble correctly, which could also explain the compensatory upregulation of specific Complex III subunits. In addition, the renal $F_{0} F_{1}-A T P a s e$ activity and ATP levels were increased during diabetes.

Conclusion: In summary, these findings show for the first time that early (and selective) inactivation of Complex-III may contribute to the mitochondrial oxidant production which occurs in the early stages of diabetes. 


\section{Background}

Renal dysfunction is a major complication that affects 20$40 \%$ of individuals with diabetes [1] and diabetic nephropathy is the most common cause of end stage renal disease (ESRD) in the United States [2]. Currently, no treatment options are available to prevent the renal complications of diabetes except for therapies which may slow the progression through intensive control of blood glucose and blood pressure [3]. Thus, it is important to identify new therapeutic targets that might lead to the prevention of diabetes-induced nephropathy.

Several reports indicate that hyperglycemia-induced generation of superoxide within the mitochondria plays a major role in the development of diabetic complications [4-7]. Nevertheless, the role that hyperglycemia has on renal mitochondrial respiratory complex function has not been thoroughly investigated. A few studies have indicated that diabetes induces alterations in the activities of mitochondrial respiratory complexes and mitochondrial respiration in the kidney $[8,9]$. Rosca et al. demonstrated that renal Complex-III was a target for glycation and inhibition during chronic diabetes (12 months) [8]. A study by Katyare and Satav showed that respiration rates and ATPase activity were elevated in diabetic renal mitochondria. This study also demonstrated that renal mitochondria were tightly coupled during diabetes [9]. In addition, Complex-III has also been shown to be inhibited during chronic diabetes in diabetic mouse retina [10]. Among the four respiratory complexes I through IV that comprise the electron transport chain, Complex-I and III are thought to be the major sources for superoxide generation within the mitochondria [11]. However, the effect that short-term hyperglycemia has on renal mitochondrial complexes has not been examined in detail, and may lead to a more precise understanding of the initiating events involved with mitochondrial oxidant production during diabetes.

In this study, we have examined the status of renal mitochondrial complexes during the early stages (5-weeks) of experimental Type-1 diabetes using the streptozotocininduced diabetes rat model. Our findings provide evidence for the first time that short-term diabetes caused selective Complex-III inactivation (with no effect on Complex I, II, or IV). In addition, further studies indicated that two Complex III subunits were induced following diabetes. These results suggest that in the early stages of hyperglycemia Complex III has assembly defects which could cause oxidant generation, leading to impairment of mitochondrial and renal function during hyperglycemia.

\section{Methods}

\section{Streptozotocin-induced diabetes model}

Male Sprague Dawley rats weighing $200 \pm 50$ g obtained from Harlan Sprague Dawley Inc. were used for this study.
All of the animal protocols were approved by the Institutional Animal Care and Use Committee at the University of Arkansas for Medical Sciences to perform as described in the paper. Rats were randomly divided into two groups: control and diabetic. Diabetes was induced by injecting overnight fasted rats with streptozotocin (STZ; $65 \mathrm{mg} / \mathrm{kg}$, i.p.) dissolved in $0.5 \mathrm{ml}$ saline, while the control rats were injected with $0.5 \mathrm{ml}$ saline. Fasting blood glucose levels were measured 48 hours post-STZ injection and rats with blood glucose levels greater than $250 \mathrm{mg} / \mathrm{dl}$ were classified as "diabetic". Diabetes-induced changes were studied at 5 weeks post-STZ injection $(n=6)$.

\section{Metabolic studies, organ harvest and blood collection}

Animals were kept in metabolic cages and acclimatized overnight prior to urine collection. Urine was collected from each rat for $24 \mathrm{hr}$, and their $24 \mathrm{hr}$ food and water intake were also measured. Animals were sacrificed under isoflurane anesthesia and their kidneys harvested. Blood was collected via intracardiac puncture and serum samples were stored at $-80^{\circ} \mathrm{C}$ until used for creatinine measurement.

\section{Creatinine clearance $\boldsymbol{\&}$ urine albumin measurement}

Serum creatinine $(\mathrm{sCr})$ and urine creatinine $(\mathrm{uCr})$ levels were measured by a modified Jaffe's method (Pointe Scientific, Canton, MI, USA) in a Cobas Mira clinical analyzer (Roche Diagnostics, Indianapolis, IN, USA). Creatinine clearance $(\mathrm{CrCl})$ was then calculated from the formula: $\mathrm{CrCl}(\mathrm{ml} / \mathrm{min} / \mathrm{kg})=(\mathrm{uCr} \times$ uVolume $\times$ Body weight $) /(\mathrm{sCr} \times 1440 \mathrm{~min} \times 1000)$. Urine albumin levels were determined by an immunoturbidometric assay (Pointe Scientific, Canton, MI, USA) in a Cobas Mira clinical analyzer and expressed as $\mathrm{mg} / \mathrm{day} / \mathrm{kg}$.

\section{Histological scoring}

Periodic acid-Schiff (PAS) staining was performed on formalin fixed, paraffin embedded kidney sections to assess tubular integrity and renal morphology as previously described $[12,13]$. Tissue sections were scored on a 0 to 4 scale by two pathologists blinded to the treatment groups according to the extent of tubular dilation and glomerular changes as follows: $0=$ absent; $1=$ mild; $2=$ moderate; 3 = severe; 4 = very severe.

\section{Measurement of respiratory complex activities}

Renal mitochondria were isolated from whole kidneys by differential centrifugation in a sucrose-containing buffer as previously described $[14,15]$. The activity of mitochondrial Complexes-I through IV as described by BirchMachin et al. [16] with minor modifications as previously described [15].

$\mathrm{F}_{\mathrm{o}} \mathrm{F}_{1}$-ATP-ase (Complex-V) activity was assayed by measuring the release of $P_{i}$ from ATP by the method of Law et 
al.[17]. Each sample was run in the presence and absence of oligomycin $(10 \mu \mathrm{g} / \mathrm{ml})$, and the $F_{o} F_{1}$-ATP-ase activity was expressed as the oligomycin-sensitive ATPase activity.

\section{Blue Native Gel Electrophoresis (BN-PAGE)}

Mitochondrial complexes were analyzed by performing BN-PAGE on the control and diabetic mitochondria as previously described [15]. To confirm the identity of mitochondrial Complex-III following BN-PAGE, the native gel was directly transferred to a PVDF membrane after first dimension and probed for known Complex III subunits Core 2 and Rieske subunits as described below. In addition, immunoblot analysis for Rieske and Core 2 subunits was performed following two-dimensional BNPAGE by performing BN-PAGE, excising the Complex III bands, followed by SDS-PAGE in the second dimension.

\section{ATP Assay}

ATP levels were determined in the mitochondrial fractions of control and diabetic rat kidneys using a luciferase based bioluminescent assay kit (Sigma-Aldrich, St. Louis, MO, USA) in a TD 20/20 luminometer (Turner Designs, Sunnyvale, CA, USA).

\section{Immunoprecipitation of Complex-III}

Complex-III was immunoprecipitated from control and diabetic rat renal mitochondria $(2.5 \mathrm{mg})$ as described by Shilling et al. [18] using Complex-III immunocapture beads (MS301) from Mitosciences, USA. After SDS-PAGE, the gels were stained with Coomassie blue staining solution $(0.1 \%$ bromophenol blue R-250 in $40 \%$ methanol and $10 \%$ acetic acid) for 30 minutes and then destained overnight in $7.5 \%$ acetic acid/5\% methanol solution.

For immunoblotting, the gel was transferred to a PVDF membrane, and probed for Core 2 protein or the Rieske subunit using mouse monoclonal antibodies - MS304 or MS305 (Mitosciences) at 1:1,000 dilution overnight at $4{ }^{\circ} \mathrm{C}$. Blots were then probed with peroxidase labeled goat anti-mouse secondary antibody, and developed using an enhanced chemiluminescent substrate system.

\section{Statistical Analysis}

Data analysis was performed using Origin 6.0. All values were expressed as mean \pm SEM. Independent t-test (twotailed) was used to compare the mean values between control and diabetic groups. Differences were considered statistically significant if the p values were less than 0.05 .

\section{Results}

Diabetic Rat Model

Rats made diabetic with STZ had fasting blood glucose levels of $350 \mathrm{mg} / \mathrm{dl}, 48$ hours after injection. Consistent with numerous reports, our diabetic rats (5-weeks postSTZ) showed a significant elevation in fasting blood glu- cose, $480.0 \pm 69.3 \mathrm{mg} / \mathrm{dl} \mathrm{Vs} 66.3 \pm 3.8 \mathrm{mg} / \mathrm{dl}$ in an agematched control group.

Diabetic rats also exhibited the typical hallmark features of diabetes: polyuria, polydipsia, polyphagia, and a significant decrease in body weight compared to the control group (Table 1).

\section{Diabetes induces hyperfiltration, albuminuria, and renal damage}

Creatinine clearance, a measure of glomerular filtration rate, was used to monitor kidney function, and urinary albumin excretion was used as a marker for early development of renal injury in our diabetic rat model. A significant elevation in creatinine clearance was observed in the diabetic group $(6.53 \pm 0.36 \mathrm{ml} / \mathrm{min} / \mathrm{kg})$ compared to the age-matched control group (4.80 $\pm 0.42 \mathrm{ml} / \mathrm{min} / \mathrm{kg})$, indicating STZ-induced diabetes caused hyperfiltration in the kidney (Figure 1A). In addition, STZ-injected rats also showed a significant increase in urinary albumin excretion $(8.43 \pm 1.74 \mathrm{mg} / \mathrm{day} / \mathrm{kg}$ in diabetic group Vs $0.9 \pm$ $0.03 \mathrm{mg} / \mathrm{day} / \mathrm{kg}$ in age-matched controls) (Figure 1B).

Periodic Acid-Schiff (PAS) staining (which stains carbohydrates in brush borders, tubules, and glomerular basement membrane) was used to assess renal damage in the diabetic rat kidneys. Histological scoring of PAS stained kidney sections by two pathologists revealed that diabetes caused a moderate renal damage (a score of 0.17 in control Vs 2.0 in diabetic group) (Figure 1C).

\section{Hyperglycemia induces specific alterations to renal mitochondrial respiratory complexes}

To examine whether early diabetes had a significant impact on the renal mitochondrial respiratory chain, we used a relatively novel technique called Blue Native-Polyacrylamide Gel Electrophoresis (BN-PAGE). BN-PAGE

Table I: Metabolic effects of STZ-induced diabetes in rats.

\begin{tabular}{lll}
\hline Parameters & Control & Diabetic \\
\hline Fasting blood glucose $(\mathrm{mg} / \mathrm{dl})$ & $66.3 \pm 3.8$ & $480.0 \pm 69.3^{*}$ \\
\hline Body weight $(\mathrm{BW})(\mathrm{g})$ & $371 \pm 9.5$ & $260.6 \pm 17.6^{*}$ \\
\hline Food Intake (g//00 g BW) & $5.7 \pm 0.3$ & $14.3 \pm 0.4^{*}$ \\
\hline Water Intake (ml/100 g BW) & $9.4 \pm 1.7$ & $73.2 \pm 3.1^{*}$ \\
\hline Urine Output (ml/100 g BW) & $3.4 \pm 0.3$ & $52.1 \pm 2.9^{*}$ \\
\hline
\end{tabular}

This table shows the effect of STZ-induced diabetes (5-week postSTZ) on fasting blood glucose, food intake, water intake, and urine output in male Sprague-Dawley rats. All values are expressed as Mean $\pm \operatorname{SEM}(n=6) . * P<0.05$ compared with age-matched (5-week) controls. 

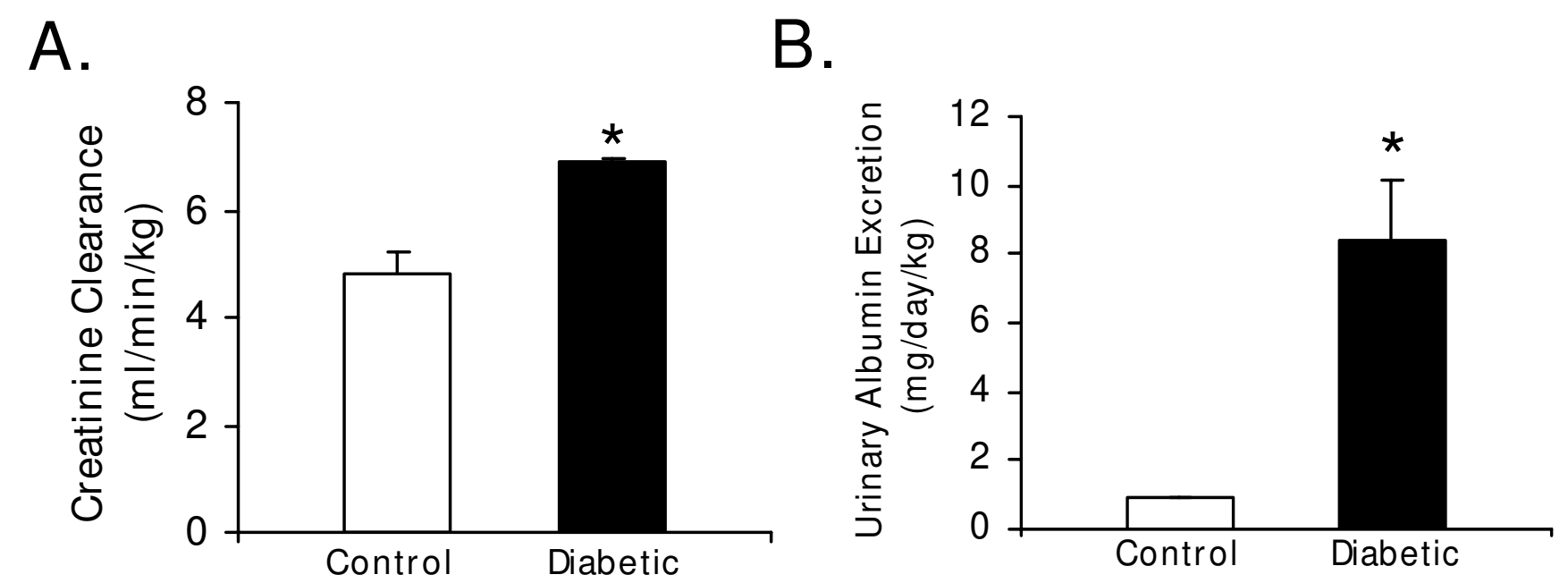

C.
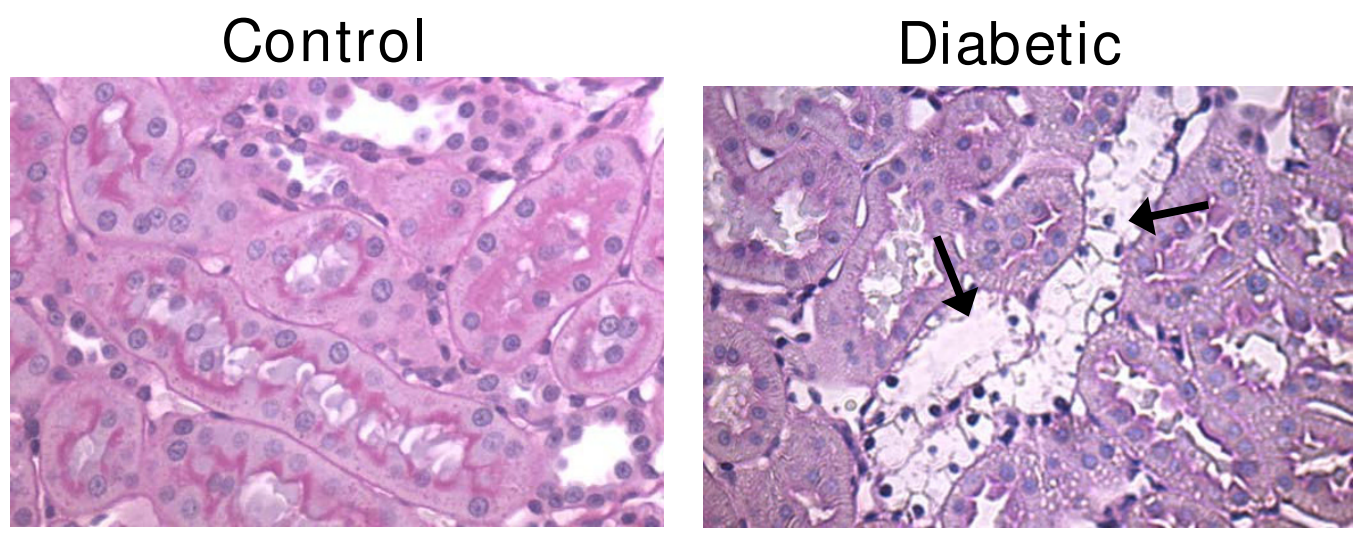

Figure I

Diabetes-induced renal dysfunction. Effect of STZ-induced diabetes (5-week post-STZ) on, A) Creatinine clearance, and B) Urinary albumin excretion in male Sprague-Dawley rats. Values are expressed as Mean \pm SEM $(n=6)$. * P < 0.05 compared with age-matched (5-week) controls. C) Periodic acid-Schiff (PAS) stained sections of control and (5-week post-STZ) diabetic rat kidneys observed under $400 \times$ magnification. Arrows indicate regions of renal damage. Experiments were repeated three times with similar results.

directly detects the membrane bound mitochondrial respiratory complexes and, thus, can pinpoint which of the five respiratory complexes (I-V) show the most intense change in levels and assembly during diabetes [19].

The BN-PAGE data indicated that Complex-III was decreased $(77.8 \%$ of control) and Complex-V was increased in the diabetic kidneys compared to the control group (Figure $2 \mathrm{~A}$ and $2 \mathrm{~B}$ ). In addition to BN-PAGE, respiratory complex activity assays were performed which further confirmed that Complex-III activity was significantly reduced in diabetic renal mitochondria $(66.32 \pm 1.93 \%)$ as compared to the age-matched controls $(100 \pm 3.58 \%$, Figure 2C); while $\mathrm{F}_{0} \mathrm{~F}_{1}$-ATPase (Complex-V) activity was increased $(148.71 \pm 6.45 \%$ in diabetic Vs $100 \pm 3.37 \%$ in control; Figure 2D). Consistent with increased $\mathrm{F}_{0} \mathrm{~F}_{1}$ -
ATPase activity, ATP levels were also significantly increased in diabetic rat kidneys $(143.39 \pm 11.56 \%)$ compared to the age-matched controls $(100 \pm 2.01 \%$; Figure $2 \mathrm{E})$. Interestingly, no significant changes were observed in the activities of Complexes I, II and IV between the diabetic and control groups (Figure 2F-H). It is important to realize that Complex III contains 11 subunits. Hence, further studies were performed to identify the specific subunits within Complex-III that might be altered during hyperglycemia using Complex III immunoprecipitation.

Surprisingly, Complex III immunoprecipitation in the diabetic samples yielded more proteins associated with Complex III when compared to control samples as visualized by Coomassie staining (Figure 3A). The precise reason for the enhanced protein staining in the diabetic 

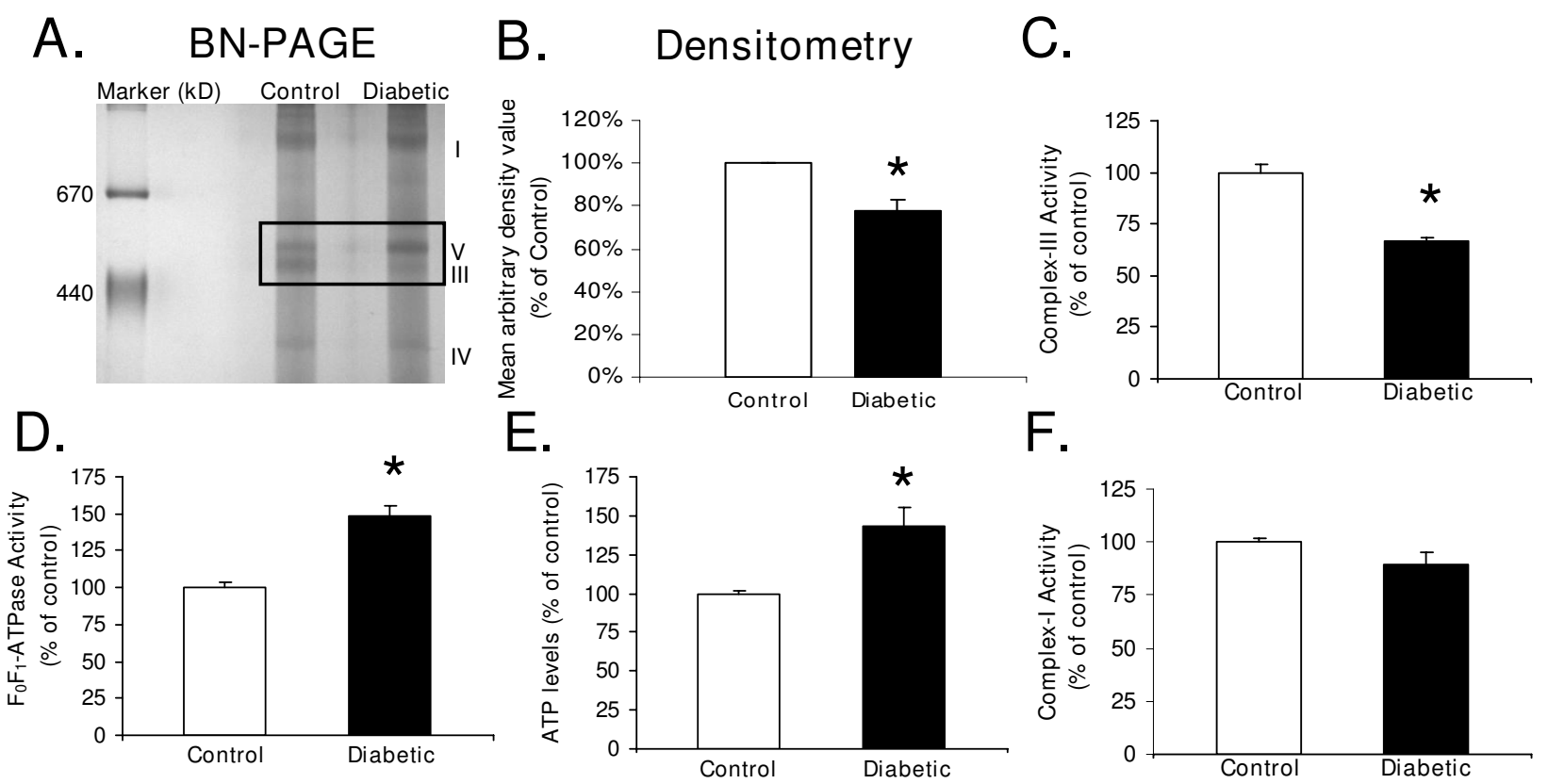

F.
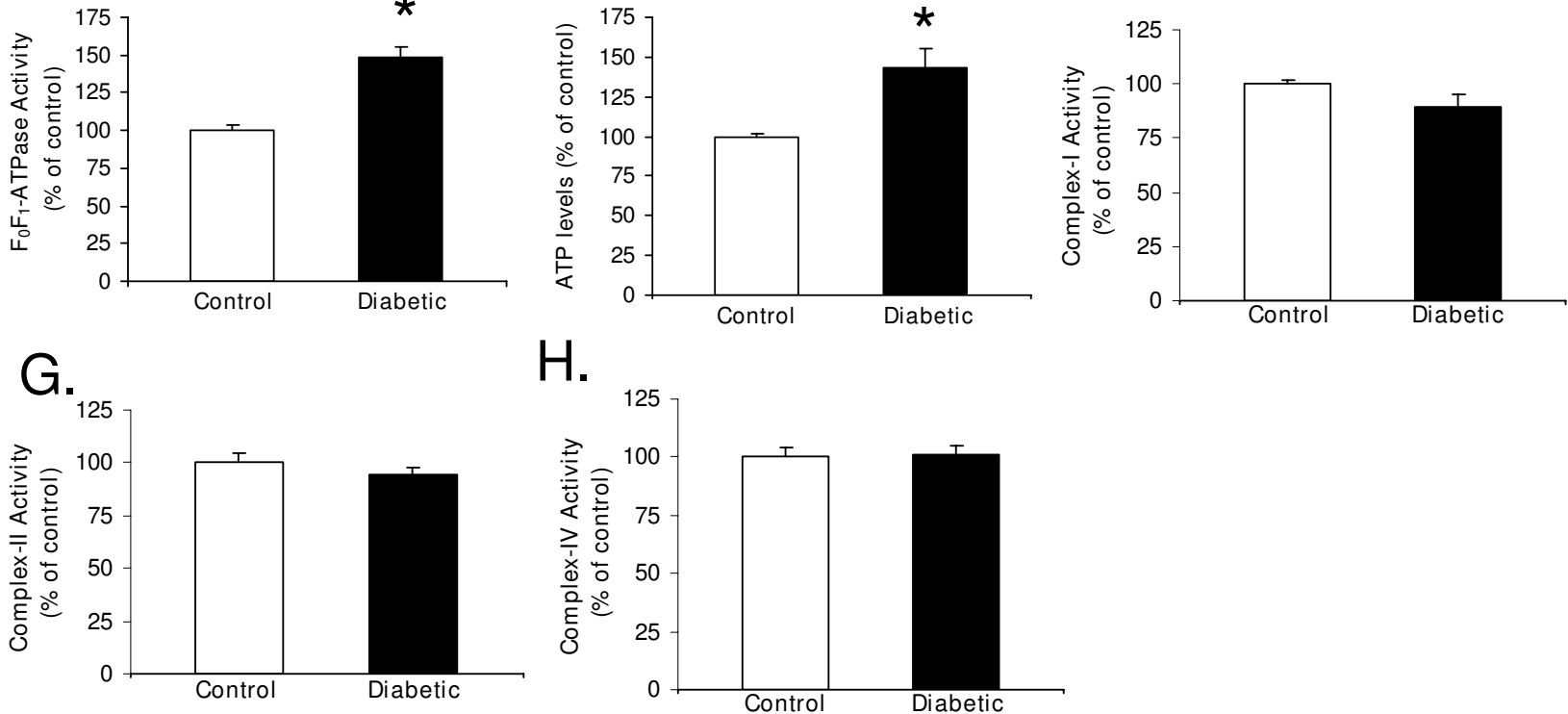

Figure 2

Diabetes-induced alterations in the activity of mitochondrial complexes and ATP levels. A) Blue Native Gel Electrophoresis (BN-PAGE) showing alterations in mitochondrial complex proteins during diabetes. The box represents Complexes-III and $\mathrm{V}$ that differ in control and diabetic (5-week post-STZ) mitochondria. B) Densitometric analysis of BN-PAGE showing a decrease in Complex III levels during diabetes. (C-H) Bar graphs indicating inactivation of mitochondrial ComplexIII (C), and induction of $F_{0}, F_{1}-A T P a s e ~(C o m p l e x-V)$ activity (D), and ATP levels (E) in diabetic rat kidney as compared to controls. Activities of renal mitochondrial complexes: F) Complex-I, G) Complex-II, and H) Complex-IV, were not altered by diabetes. All values are expressed as percentage Mean \pm SEM $(n=5)$ of controls (set to I00). * $\mathrm{P}<0.05$ compared with agematched controls.

mitochondria remains unclear, but may be related to altered assembly of Complex III. Nevertheless, we chose to focus on the key redox sensitive subunit within Complex III, the Rieske protein as well as the Core 2 protein, a subunit that is not directly involved with electron transfer.

Thus, Western blot analysis was performed for Rieske or Core 2 protein following Complex III immunoprecipitation. Diabetes appeared to increase Complex III associated Rieske and Core 2 proteins when compared to control kidneys (Figure 3B and 3C). The increased expression of specific Complex III subunits was further con- firmed by Western blot analysis using renal mitochondria (without immunoprecipitation) which also showed an upregulation of the Complex-III subunits, Rieske (133.33 $\pm 3.1 \%)$ and Core 2 protein $(136.69 \pm 1.44 \%)$ during diabetes (Figure 3D and 3E) as compared to control. GAPDH was used as the loading control.

Additional experiments were carried out to verify the identity of Complex III following BN-PAGE. These included Western blotting for Rieske and Core 2 proteins after BN-PAGE (Figure 4A \&4B) as well as BN-PAGE followed by SDS-PAGE in the second dimension (2D BN- 

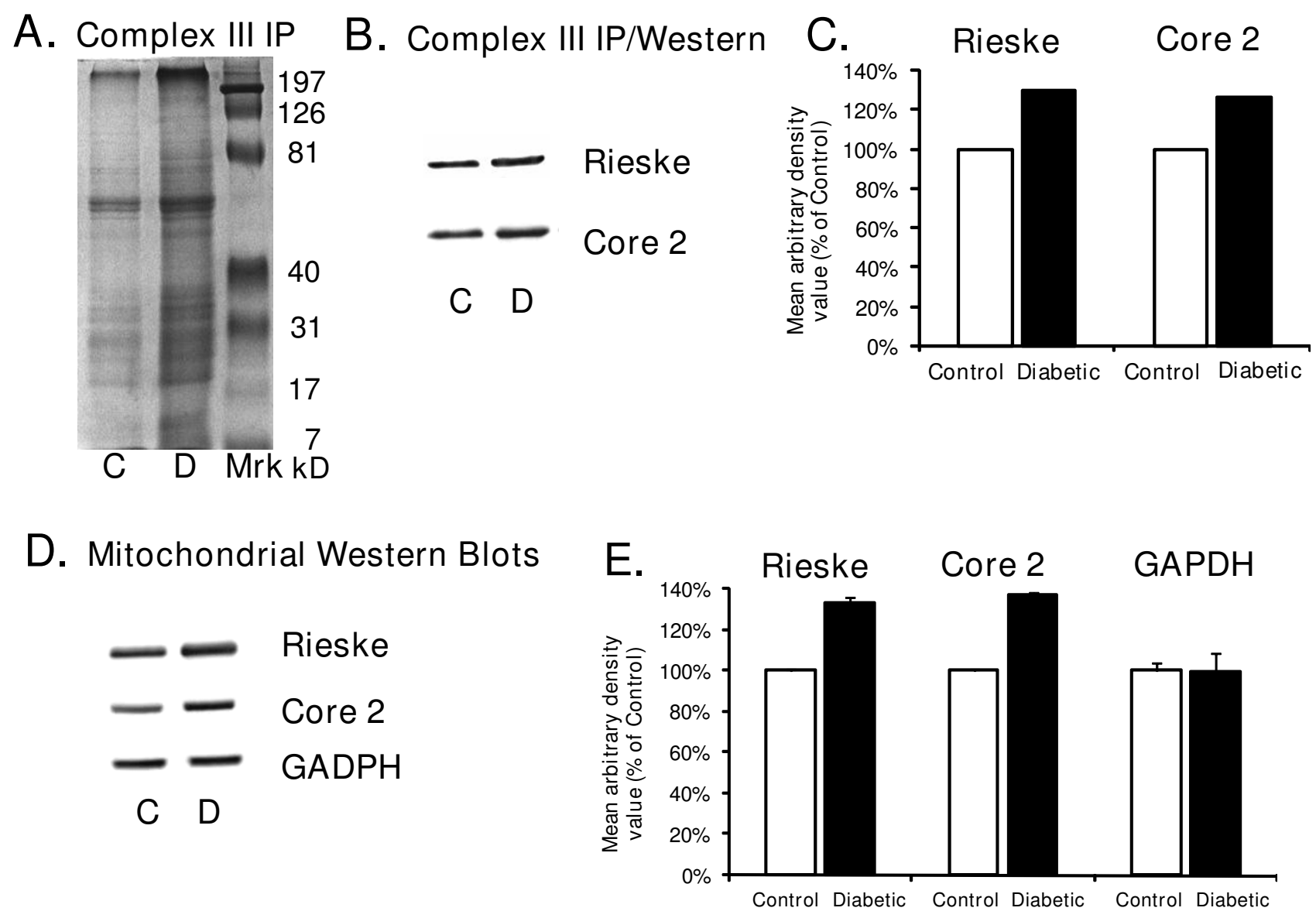

Figure 3

Diabetes-induced upregulation of renal Complex-III subunits, Rieske protein and Core 2 protein. A) Coomassie blue stained SDS-Polyacrylamide gel (following immunoprecipitation of Complex-III in control (C) and diabetic (D) rat kidney mitochondria) revealing upregulation of mitochondrial Complex-III subunit proteins during diabetes. B) Complex III IP followed by Western blot analysis revealed upregulation of Complex III associated Rieske Protein and Core 2 Protein subunits during diabetes. C) Densitometric analysis showing Complex III associated subunits were upregulated during diabetes. D) Rieske Protein and Core 2 Protein Western blot analysis of total mitochondria. GAPDH Western blot analysis was used as a loading control. E) Densitometric analysis of the Western blot shown in panel D. Blots are representative of three separate experiments.

PAGE) (Figure 4C \&4D). These data clearly demonstrated that the band identified as Complex III in the BN-PAGE contains both the Rieske and Core 2 subunits of Complex III.

\section{Discussion}

These results indicate that renal Complex-III appears to be an early and specific mitochondrial target during experimental type-1 diabetes. Complex-III is centrally located in the electron transfer process and has been implicated as one of the major sites for superoxide generation in the mitochondria during diabetes $[8,10]$. Partial inhibition of Complex-III during conditions of increased respiration
[9] would decrease the transfer of electrons from ubiquinol to Complex-III, and increase the half-life of ubisemiquinone, which leads to generation of superoxide $[8,10]$.

Intriguingly, our results indicated an upregulation of renal Complex-III subunits, Core 2 and Rieske protein during diabetes. However, using BN-PAGE it was shown that Complex III was not correctly assembled (decreased levels on BN-PAGE) which also correlated with a decrease in Complex-III activity. Thus, the paradoxical increase in expression of individual subunits, Core 2 and Rieske proteins could be a compensatory response to restore the activity of Complex-III during diabetes. 


\section{BN-PAGE/Western Blot}

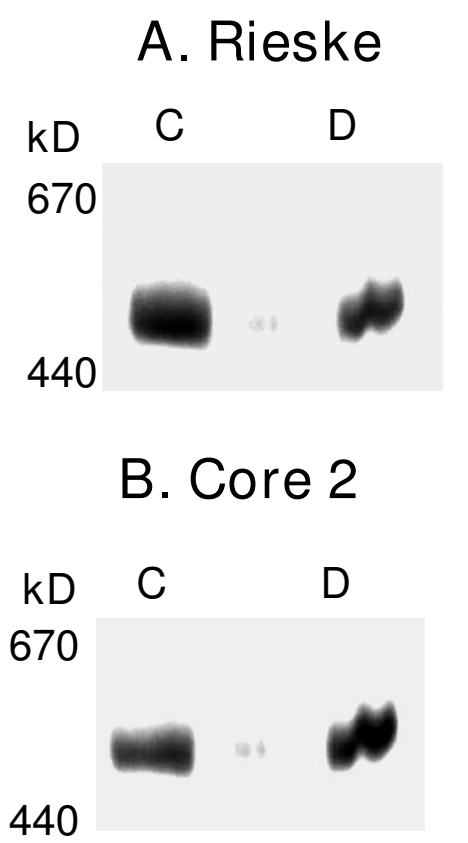

\section{D-BN-PAGE/Western Blot}
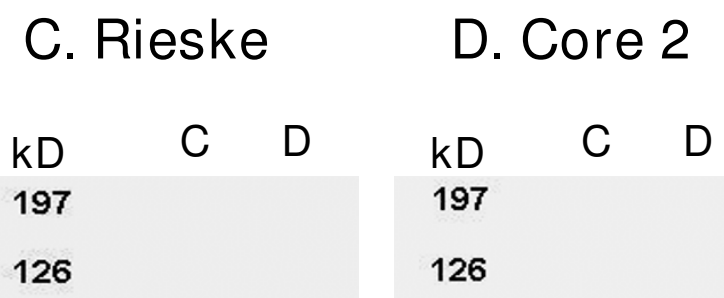

81

81

40

40

31

17

17

\section{Figure 4}

Identification of renal Complex-III following BN-PAGE. Western blot analysis for Rieske Protein (A and C) and Core 2 Protein (B and $\mathbf{D}$ ) to confirm the identity of the Complex-III band after one dimension BN-PAGE (left panel/A and B) and 2D-BN-PAGE (right panel/C and D) using control (C) and diabetic (D) rat kidney mitochondria.

Rieske subunit is a nuclear encoded protein that is imported into the mitochondrial matrix where it undergoes a two-step cleavage of its pre-sequence and is translocated into the inner mitochondrial membrane. It has an iron-sulfur [2Fe-2S] redox center that centrally participates in the $\mathrm{Q}$ cycle by transferring an electron from ubiquinol to cytochrome $c_{1}$, which then donates it to cytochrome c. Core 2 subunit, which is also a nuclear encoded protein is a homolog of mitochondrial processing protease-alpha subunit and is thought to be important for mitochondrial protein import, processing and integrity of Complex-III [20]. Although, Core 2 subunit is not primarily involved in electron transfer, genetic deletion of Core 2 has been shown to affect the assembly of ComplexIII [21]. Thus, an increase in the Rieske as well as the Core 2 subunits suggests a possible assembly defect in Complex-III.

To our knowledge, this is the first report demonstrating that renal Complex-III and its subunits are altered in the early stages of diabetes. A study by Rosca et al. showed previously that Complex-III was a target for glycation by methyl glyoxal and inactivated during chronic diabetes (12 months) [8], while no significant changes were reported at 2 months of diabetes. Although, the reason for this discrepancy remains unknown; one difference could be the fraction of the kidney analyzed in the two studies. Our study utilized whole rat kidneys, whereas the study by Rosca et al. used the cortical fractions of kidneys. Although the renal cortex has been primarily considered to be the energetically active segment of the nephron, several studies suggest that the medullary thick ascending limb (mTAL) is an active site for reabsorption in kidney and thus might play a major role in mitochondrial superoxide production. In addition, increased oxidant production has been observed in both the cortical and medullar regions of the kidney. Thus, we felt it was important to include both cortex and medulla (whole kidney) for mitochondrial isolation and study the net effect of diabetes on the renal mitochondria.

Despite the findings by Katyare and Satav [9] who showed increased cytochrome aa3 contents in renal mitochondria during diabetes, we did not detect any significant changes in Complex IV activity in our rat model of diabetes. It is also important to note that the authors could not correlate the changes in cytochrome content with the altered respiration rates observed during diabetes. In addition, these 
authors did not measure Complex IV activity and we did not measure cytochrome aa3 contents so a direct comparison of these studies is difficult to assess. Again, one important difference between the two studies is the source of renal mitochondria used: we used whole kidney mitochondria, while Katyare and Satav used cortical mitochondria as discussed above.

Our results also showed that $\mathrm{F}_{0} \mathrm{~F}_{1}$-ATPase activity and renal ATP levels were significantly increased in the diabetic renal mitochondria compared to controls. Interestingly, the study by Katyare and Satav [9] also demonstrated that respiration and ATPase activity were increased in diabetic renal mitochondria. Thus, it is possible that the increased respiration leads to increased proton motive force (hyperpolarization) and increased ATP levels which serve to fulfill the increased energy demands of the kidney during diabetes. Concomitantly, the increased respiration would likely result in more superoxide (oxidant) generation which could lead to alterations in Complex III. In fact, we have observed that renal proximal tubule cells exposed to hyperglycemia $(25 \mathrm{mM} ; 48$ hr) results in mitochondrial hyperpolarization, ComplexIII inhibition, and oxidant production (manuscript under review).

\section{Conclusion}

In conclusion, we have demonstrated that early stages of diabetes induced alterations in Complex-III, increased ATP synthase activity, and renal dysfunction. These diabetes-induced alterations in activities of mitochondrial complexes and energy status could contribute to the underlying role of oxidative stress in the pathogenesis of diabetic nephropathy. Our future in vitro studies will explore the mechanistic pathways by which hyperglycemia induces mitochondrial complex dysfunction and oxidant production. Finally, strategies to limit the extent of renal mitochondrial damage during hyperglycemia (by therapeutic agents that will specifically modulate mitochondrial function) might prevent or inhibit the development of nephropathy in the diabetic population.

\section{Abbreviations}

BN-PAGE: Blue Native Polyacrylamide Gel Electrophoresis; Complex-III: Ubiquinol:Cytochrome c oxidoreductase or Cytochrome bc1 complex; $\mathrm{CrCl}$ : Creatinine Clearance; ESRD: End Stage Renal Disease; GAPDH: Glyceraldehyde3-Phosphate Dehydrogenase; PAS: Periodic acid-Schiff; sCr: Serum Creatinine; STZ: Streptozotocin; uCr: Urine Creatinine.

\section{Competing interests}

The authors declare that they have no competing interests.

\section{Authors' contributions}

SM and LM were responsible for the overall design of the study. SM performed most of the biochemical analyses and renal function studies. TM and HS provided technical guidance and advice for the biochemical methods. JM performed the histological assessment. JM, RB and LM critically reviewed the manuscript, and played an important role with regard to intellectual content. SM wrote the draft of the manuscript. All the authors have read and approved the final manuscript.

\section{Acknowledgements}

We thank Drs. John P. Crow and Philip R. Mayeux for their valuable comments and discussion.

This research study was funded by a Pilot grant from University of Arkansas for Medical Sciences, Little Rock, AR.

\section{References}

I. Gross JL, de Azevedo MJ, Silveiro SP, Canani LH, Caramori ML, Zelmanovitz T: Diabetic nephropathy: diagnosis, prevention, and treatment. Diabetes Care 2005, 28: I64-176.

2. U.S. Renal Data System: USRDS 2008 Annual Report, in Atlas of EndStage Renal Disease in the United States, Bethesda, MD, National Institutes of Health, National Institute for Diabetes and Digestive and Kidney Diseases 2008.

3. Cooper ME, Jandeleit-Dahm K, Thomas MC: Targets to retard the progression of diabetic nephropathy. Kidney Int 2005, 68:1439-1445.

4. Brownlee M: Biochemistry and molecular cell biology of diabetic complications. Nature 200I, 4 |4:8I3-820.

5. Du XL, Edelstein D, Rossetti L, Fantus IG, Goldberg H, Ziyadeh F, Wu J, Brownlee M: Hyperglycemia-induced mitochondrial superoxide overproduction activates the hexosamine pathway and induces plasminogen activator inhibitor-I expression by increasing Sp I glycosylation. PNAS 2000, 97: I 2222- I 2226.

6. Nishikawa T, Edelstein D, Brownlee M: The missing link: a single unifying mechanism for diabetic complications. Kidney Int Suppl 2000, 77:S26-S30.

7. Nishikawa T, Edelstein D, Du XL, Yamagishi S, Matsumura T, Kaneda Y, Yorek MA, Beebe D, Oates PJ, Hammes HP, et al.: Normalizing mitochondrial superoxide production blocks three pathways of hyperglycaemic damage. Nature 2000, 404:787-790.

8. Rosca MG, Mustata TG, Kinter MT, Ozdemir AM, Kern TS, Szweda LI, Brownlee M, Monnier VM, Weiss MF: Glycation of mitochondrial proteins from diabetic rat kidney is associated with excess superoxide formation. Am J Physiol Renal Physiol 2005, 289:F420-F430.

9. Katyare SS, Satav JG: Effect of streptozotocin-induced diabetes on oxidative energy metabolism in rat kidney mitochondria. A comparative study of early and late effects. Diabetes Obes Metab 2005, 7:555-562.

10. Kanwar M, Chan PS, Kern TS, Kowluru RA: Oxidative damage in the retinal mitochondria of diabetic mice: possible protection by superoxide dismutase. Invest Ophthalmol Vis Sci 2007, 48:3805-38II.

II. Chen Q, Vazquez EJ, Moghaddas S, Hoppel CL, Lesnefsky EJ: Production of reactive oxygen species by mitochondria: central role of complex III. J biol Chem 2003, 278:36027-3603I.

12. Cruthirds DL, Novak L, Akhi KM, Sanders PW, Thompson JA, MacMillan-Crow LA: Mitochondrial targets of oxidative stress during renal ischemia/reperfusion. Arch Biochem Biophys 2003, 412:27-33.

13. Saba H, Batinic-Haberle I, Munusamy S, Mitchell T, Lichti C, Megyesi J, MacMillan-Crow LA: Manganese porphyrin reduces renal injury and mitochondrial damage during ischemia/reperfusion. Free Radic Biol Med 2007, 42: I57I-I578.

14. MacMillan-Crow LA, Crow JP, Kerby JD, Beckman JS, Thompson JA: Nitration and inactivation of manganese superoxide dis- 
mutase in chronic rejection of humanárenaláallografts. PNAS 1996, 93: II853-II858.

15. Saba H, Munusamy S, MacMillan-Crow LA: Cold preservation mediated renal injury: involvement of mitochondrial oxidative stress. Ren Fail 2008, 30:125-133.

16. Birch-Machin MA, Turnbull DM: Assaying mitochondrial respiratory complex activity in mitochondria isolated from human cells and tissues. Methods Cell Biol 200I, 65:97-II7.

17. Law RH, Manon S, Devenish RJ, Nagley P: ATP synthase from Saccharomyces cerevisiae. Methods Enzymol 1995, 260:133-163.

18. Schilling B, Murray J, Yoo CB, Row RH, Cusack MP, Capaldi RA, Gibson BW: Proteomic analysis of succinate dehydrogenase and ubiquinol-cytochrome c reductase (Complex II and III) isolated by immunoprecipitation from bovine and mouse heart mitochondria. Biochim Biophys Acta 2006, I762:213-222.

19. Van Coster R, Smet J, George E, De Meirleir L, Seneca S, Van Hove J, Sebire G, Verhelst H, De Bleecker J, Van Vlem B, et al.: Blue native polyacrylamide gel electrophoresis: a powerful tool in diagnosis of oxidative phosphorylation defects. Pediatr Res 200I, 50:658-665.

20. Saraste $M$ : Oxidative Phosphorylation at the fin de si\&egrave;cle. Science 1999, 283: | 488- 1493.

21. Oudshoorn P, Van Steeg H, Swinkels BW, Schoppink P, Grivell LA: Subunit II of yeast QH2:cytochrome-c oxidoreductase. Nucleotide sequence of the gene and features of the protein. Eur J Biochem 1987, 163:97-103.

\section{Pre-publication history}

The pre-publication history for this paper can be accessed here:

http://www.biomedcentral.com/1472-6823/9/2/prepub

Publish with Biomed Central and every scientist can read your work free of charge

"BioMed Central will be the most significant development for disseminating the results of biomedical research in our lifetime. "

Sir Paul Nurse, Cancer Research UK

Your research papers will be:

- available free of charge to the entire biomedical community

- peer reviewed and published immediately upon acceptance

- cited in PubMed and archived on PubMed Central

- yours - you keep the copyright

Submit your manuscript here:

http://www.biomedcentral.com/info/publishing_adv.asp 